\title{
Geo-epidemiological reporting and spatial clustering of the 10 most prevalent cancers in Iran
}

\author{
Ebrahim Babaee, ${ }^{1}$ Gholamreza Roshandel, ${ }^{2,3}$ Meysam Olfatifar, ${ }^{4}$ Arash Tehrani-Banihashemi, ${ }^{1}$ \\ Arezou Ashaari, ${ }^{1}$ Marzieh Nojomi ${ }^{1,5}$ \\ ${ }^{1}$ Department of Community Medicine, Preventive Medicine and Public Health Research Centre, Iran \\ University of Medical Sciences, Tehran, Iran; ${ }^{2}$ Golestan Research Centre of Gastroenterology and \\ Hepatology, Golestan University of Medical Sciences, Gorgan, Iran; ${ }^{3}$ Iranian National Population-Based \\ Cancer Registry Secretariat, Cancer Office, Deputy of Health, Ministry of Health, Tehran, Iran; \\ ${ }^{4}$ Gastroenterology and Liver Diseases Research Centre, Research Institute for Gastroenterology and Liver \\ Diseases, Shahid Beheshti University of Medical Sciences, Tehran, Iran; ${ }^{5}$ Department of Sociology \& \\ Anthropology, Nipissing University, North Bay, ON, Canada
}

\begin{abstract}
Cancer is a problem of both global and local concern. We determined the geo-epidemiological and spatial distribution of the 10 most common cancers in Iran. We used the data of the Iranian Cancer Registry for the year 2014 analysing the prevalence of 112,131 registered cancer cases with the aim of detecting potential
\end{abstract}

Correspondence: Marzieh Nojomi, Preventive Medicine and Public Health Research Center, Psychosocial Health Research Institute, Community and Family Medicine Department, School of Medicine, Iran University of Medical Sciences, Postal code: 1449614542, Tehran, Iran. Fax: +98.2186703350.

E-mail: mnojomi@iums.ac.ir

Key words: Cancer; epidemiology; spatial analysis; cluster analysis; Iran.

Acknowledgements and funding: this research was supported by the Elite Researcher Grant Committee under award number of 982858 from the National Institute for Medical Research Development (NIMAD), Tehran, Iran. We would like to thank the Iranian national populationbased cancer registry secretariat and Cancer Office of the Ministry of Health of Iran for their complete collaborations.

Conflict of interests: the corresponding author reports grants from the National Institutes for Medical Research Development (NIMAD), during performing the study. All the other authors declare no conflict of interests.

Received for publication: 7 June 2020.

Revision received: 12 November 2020.

Accepted for publication: 15 November 2020.

(c) Copyright: the Author(s), 2021

Licensee PAGEPress, Italy

Geospatial Health 2021; 16:904

doi:10.4081/gh.2021.904

This article is distributed under the terms of the Creative Commons Attribution Noncommercial License (CC BY-NC 4.0) which permits any noncommercial use, distribution, and reproduction in any medium, provided the original author(s) and source are credited. geographical underlying causes. The geographic distribution of cancers is reported as standardized incidence rates at the provincial level considering risk with respect to sex and age. A geographical information systems (GIS) approach based on Anselin Local Moran's index method was used to map clusters and spatial autocorrelation patterns. The mean age of the patients was 55.6 $( \pm 17.8)$ and $61.7( \pm 18.2)$ for females and males, respectively, in the database which showed $46.1 \%(n=51,665)$ of all cases to be female. Analysis of the spatial distribution of cancers showed significant differences among the different provinces. Stomach and breast cancers were the most prevalent cancers in men and females, respectively. The highest incidence rates of stomach cancer were found in Ardabil and Zanjan provinces, with 48.38 and 48.08 per 100,000 population, respectively, while Tehran and Yazd provinces had the highest incidences of breast cancer, 51.0 and 47.5 per 100,000 population, respectively. Strong clustering patterns for stomach and breast cancers were identified in the north-western provinces and in Semnan Province, respectively. These patterns indicate a diversity of geo-epidemiological contributing factors to cancer incidence in Iran.

\section{Introduction}

Cancer is a universal public health burden lacking a common pre-emptive approach. The global burden of disease study in 2017 (GBD 2017) reported 24.5 million incident cancer cases worldwide (Global Burden of Disease Cancer Collaboration, 2019). Bray et al. (2018) in their study refer to GLOBOCAN, which is another body providing estimates of cancer incidence, and report that the incidence rates of cancer and the related deaths in Asia are $48.4 \%$ and $57.3 \%$, respectively (The Lancet, 2018). According to previous studies, in developed countries, the prevalence of cancers is predicted to reach $45 \%$ by the year 2025 (Kamangar et al., 2006). Notably, in low- and middle-income countries, population growth and aging are the major contributors to the increase of cancer incidence (Global Burden of Disease Cancer Collaboration, 2019). Besides, more than $70 \%$ of all the cancer-related deaths occur in countries whose populations have a medium to the lowlevel standard of living as well as limited resources for the prevention, diagnosis, and treatment of cancer (Mousavi et al., 2008).

In Iran, aging and increased related risk factors are known as 
the main causes of the increased incidence of cancer, which is the third leading cause of death in Iran after coronary heart disease and accidents (Alireza et al., 2005; Mousavi et al., 2009). Given the importance of cancer control, effective strategies should be implemented by the health systems. Population-based cancer registries play an important role in monitoring trends related to cancer incidence, prevalence and mortality over time among different areas and social groups. Although the data presented are accurate, it is a difficult method that needs a strong structure. In 2010, Iran established the Iranian National Population-based Cancer Registry (INPCR) in order to collect and aggregate regional cancer data (Roshandel et al., 2019). This system for cancer information collection and surveillance is comprehensive, but it is equally important that the data needed can be reported and retrieved in a practical way.

Cancer maps could help to determine the regions with the highest density of cancer cases and also facilitate the investigation of environmental risk factors of specific cancer types in different regions according to the Guide for Effective Programs, publicised by the World Health Organization (WHO) (https://www.who.int/ cancer/modules/en/). Geographical information systems (GIS) can perform these goals well (Scholten et al., 1991) through applications that have since developed in different directions (Bergquist \& Manada, 2019).

Notably, creating geo-referenced maps is necessary to obtain valuable information about cancer cases concerning frequency of spatial features (Yomralioglu et al., 2009). In this regard, spatial analyses help to generate new hypotheses. However, it is also important that clusters associated with certain cumulative risks such as income, urbanization and dietary patterns or areas that require the targeted interventions can be found (Bafandeh \& Farhang, 2009; Hao et al., 2011).

So far, few studies have been conducted on this subject in Iran and those available were carried out at the provincial level and only on some types of cancer (Chamanparaa et al., 2015). In this study, we report the results of the demographical and geographical distributions of the 10 most prevalent cancers (TMPC) in Iranian men and women. We also investigated spatial clustering and autocorrelation patterns of the TMPCs (with regard to both genders) among the provinces of Iran to answer the question whether neighbouring provinces tend to have similar incidence rates and whether they also form clusters based on such correlations.

\section{Materials and methods}

\section{Study area}

This geographical registry-based study was conducted in 2020 in Iran. Located in the western part of Asia bordering the Caspian Sea, Persian Gulf and the Gulf of Oman, the country consists of 31 provinces (Figure 1) that have shown a great variability in cancer incidence, in particular with reference to the type of cancer.

\section{Data source and rates}

We used data for 2014 from INPCR that were released in 2019 (Roshandel et al., 2019). Our study covered only 30 of Iran's 31 provinces since information from Qom Province was unavailable. Still, the data covered $98 \%$ of the Iran's population, men and women, and out of 112,131 registered cases in total, we only excluded data from 216 patients due to incomplete information.

The $3^{\text {rd }}$ edition of the International Classification of Diseases for Oncology (ICD-O-3) was applied (Fritz, 2013) for tumour coding. Using demographic and geographical information, the patients' place of residence were specified and then recorded in the dataset that showed the geographic distribution of the cancers reported as age-standardized incidence rates (ASRs) at the provincial and sex and age subgroup levels. Based on the latest census published in 2011 by Iran's statistics centre, the population of Iran was considered as the reference population, with the world's standard population applied to estimate the ASRs. All provincial, age and sex specific incidence rates were expressed per 100,000 or 1,000,000 population (Segi, 1960).

\section{Geospatial analysis}

For geo-reporting we used the geographic map of Iran in shape-file format. This file was obtained from the national cartographic centre of Iran (https://www.ncc.ir/en-us/NCCPortal/1/ page/HomePage). The geographic reports of cancer cases were based on three sections. Firstly, we generally symbolized the geodemographical distributions of the TMPCs in separate maps in terms of the participants' gender using quantities expressed by gradually shaded colours. The incidence rates were reported in 5 categories on the maps based on quantities based the natural breaks (Jenks) classification method. In order to map clusters and spatial autocorrelation patterns, we applied the Anselin Local Moran's index method. For the conceptualization of the spatial relationships we selected the fixed distance band option, which imposes a sphere of influence where each feature is analysed within the context of the neighbouring features located within the distance specified by the threshold distance (distance band). For analysing the obtained data, we used Stata software version 14 (Stata Corp, College Station, TX, USA) and Microsoft Excel Worksheet. The ArcGIS software, v.10.2.2 (ESRI, Redlands, CA, USA) was applied to combine the data and produce the spatial analysis. All these analyses were completed in the ArcMap environment.

\section{Results}

The mean age of all patients (female and male) were 55.6 $( \pm 17.8)$ and $61.7( \pm 18.2)$ years, respectively, and the female share of the cancer cases investigated were $46.1 \%(n=51,665)$. Those being 75 years old or older constituted the largest age group $(n=24,446,21.8 \%)$, while the youngest group ( $\leq 4$ years) only included 952 of the cases $(0.8 \%)$. Cancer of the stomach $(n=7149)$ and breast $(n=13,120)$ were the most prevalent cancers among Iranian men and women, respectively. The order of all the most common cancers for each sex is reported in Table 1. In Tables 2 and 3, we report the ASRs as well as the sex-standardized incidence rates (per 1,000,000) of the TMPCs in men and women, respectively. The male incidence rate of stomach cancer was 1.1 and 2393.4 in the age groups $0-4$ and $\geq 75$ years, respectively, while the female incidence rate of breast cancer was 0.2 and 758.9 in the same age groups. Notably, the incidence of all cancers in both sexes increased with age, except for uterus, breast and ovarian cancer. In these three cancers, the highest incidence rates were found to be between the ages of 50 and 70 years, with a decreasing trend beginning from the age of 70 years. In men, cancer of the prostate 
amounted to $10.1 \%(\mathrm{n}=5946)$ and colorectal cancer to $9.6 \%$ $(n=5648)$, while in women colorectal cancer was $8.6 \%(n=4220)$ and stomach cancer $6.8 \%(\mathrm{n}=3348)$; these were the second and third most prevalent cancers overall, respectively.

\section{Geographical distribution and autocorrelation}

The geographical distribution of the standardized incidence rates of TMPCs for men and women at the provincial level are shown in Figures 2 and 3, respectively. Based on these results, Ardabil $(48.38$ per 100,000$)$ and Zanjan $(48.08$ per 100,000$)$ were the provinces with the highest incidence rates of stomach cancer in men occupying the first and the second rank, respectively. Among women, Tehran $(51.0$ per 100,000$)$ and Yazd $(47.5$ per 100,000$)$ were the provinces with the highest incidence rates of breast cancer. Additionally, colorectal and stomach cancers were the second and third most prevalent cancers in Iranian women. Moreover, colorectal cancer was mainly prevalent in the provinces Semnan and Tehran. Notably, Ardabil, Zanjan, East Azerbaijan and West Azerbaijan had the highest incidence rates of stomach cancer.

When we turned our attention to the spatial cluster patterns of all cancers under study for both genders, the high and low cluster points produced by the Moran's $I$ showed different distribution patterns depending on cancer type in the different provinces. For men, stomach cancer was significantly clustered with high-high clustering patterns in West Azerbaijan, East Azerbaijan, Ardabil and Zanjan provinces (Figure 4). However, we observed no such pattern for prostate cancer, but high-high clusters of larynx cancer were seen in North Khorasan and Razavi Khorasan in north-eastern Iran and a low-low pattern in Hamadan and Lorestan in the western part of the country (Figure 4). With regard to women, we observed lowlow clusters of breast cancer in Ardabil and Kurdistan and highhigh ones in Semnan, Isfahan and Yazd, which are Iran's central provinces (Figure 5). For uterus cancer, we identified a low-low cluster pattern in Ilam Province and an outlier in Zanjan Province
(Figure 5). The results of Local Moran's I for all the different cancers among men and women are shown in Figures 4 and 5.

Table 1. The most common cancers in Iranian males and females 2014.

\begin{tabular}{llcc} 
& Type of cancer & Number & Percent \\
Male & Stomach & 7149 & 12.10 \\
& Prostate & 5946 & 10.06 \\
Colorectal & 5648 & 9.56 \\
Bladder & 4825 & 8.16 \\
Lung & 4217 & 7.14 \\
Leukaemia & 2876 & 4.87 \\
Brain, nervous system & 2392 & 4.05 \\
Oesophagus & 2177 & 3.68 \\
Non-Hodgkin* & 1981 & 3.35 \\
Larynx & 1417 & 2.40 \\
Other sites & 20,477 & 34.64 \\
Total & 59,102 & 100.00 \\
Breast & 13,120 & 26.75 \\
Colorectal & 4220 & 8.60 \\
Stomach & 3348 & 6.83 \\
Thyroid & 2911 & 5.94 \\
Leukaemia & 1879 & 3.83 \\
Lung & 1839 & 3.75 \\
Brain, nervous system & 1800 & 3.67 \\
Oesophagus & 1778 & 3.63 \\
Uterus & 1726 & 3.52 \\
Ovary & 1653 & 3.37 \\
Other sites & 14,773 & 30.12 \\
Total & 49,047 & 100.00 \\
\hline
\end{tabular}

*Non-Hodgkin lymphoma.

Table 2. Standardized age-sex specific incidence rates (per 1,000,000) of the most common cancers in Iranian males 2014.

\begin{tabular}{|c|c|c|c|c|c|c|c|c|c|c|}
\hline Age group & Stomach & Prostate & Colorectal & Bladder & Lung & Leukaemia & $\begin{array}{l}\text { Nervous } \\
\text { system* }\end{array}$ & Oesophagus & $\begin{array}{c}\text { Non-Hodgkin } \\
\text { lymphoma }\end{array}$ & Larynx \\
\hline $0-4$ & 1.1 & 0.3 & 0.3 & 0.3 & 0.8 & 59.2 & 19.5 & 0 & 6.8 & 0 \\
\hline $5-9$ & 0.6 & 0.3 & 0.3 & 1.2 & 1.2 & 62.7 & 20.4 & 0.3 & 11.9 & 0 \\
\hline $10-14$ & 0.7 & 0 & 0.7 & 0.7 & 1.4 & 39.2 & 14.8 & 0 & 11.7 & 0 \\
\hline $15-19$ & 1.1 & 0.7 & 5.4 & 3.9 & 3.6 & 46.3 & 19.4 & 0 & 16.2 & 0.4 \\
\hline $20-24$ & 7.7 & 1.5 & 8.7 & 4.9 & 7.1 & 36.8 & 27.5 & 0.9 & 22.2 & 0.3 \\
\hline $25-29$ & 13,0 & 1.7 & 19.8 & 7.2 & 5.8 & 27 & 30.9 & 2.4 & 24.6 & 1.0 \\
\hline $30-34$ & 18.2 & 1.4 & 32.7 & 13.6 & 13.1 & 24.2 & 35.7 & 4.1 & 23.5 & 2.1 \\
\hline $35-39$ & 33.3 & 2,0 & 58.5 & 22.4 & 14.3 & 31.9 & 45.6 & 10.1 & 31.6 & 4.2 \\
\hline $40-44$ & 72.1 & 7.8 & 89.9 & 46.2 & 42.3 & 41.6 & 60.8 & 19.5 & 45.5 & 17.1 \\
\hline $45-49$ & 124.3 & 20.4 & 145.0 & 92.9 & 83.5 & 56.6 & 70.1 & 37.9 & 55.0 & 38.7 \\
\hline $50-54$ & 259.7 & 109.8 & 272.3 & 212.6 & 183.2 & 86.1 & 105.3 & 70.4 & 90.1 & 85.0 \\
\hline 55-59 & 438.3 & 291.6 & 415.6 & 358.1 & 285.0 & 129.9 & 143.7 & 122.2 & 120.4 & 135.9 \\
\hline $60-64$ & 642.6 & 629.1 & 604.3 & 539.7 & 459.8 & 174.8 & 182.0 & 196.4 & 154.1 & 182 \\
\hline $65-69$ & 1128.7 & 1131.2 & 743.0 & 728.1 & 666.3 & 241.1 & 223.8 & 336.3 & 185.4 & 225 \\
\hline $70-74$ & 1630.2 & 1735.2 & 1132.9 & 1003.3 & 882.5 & 302.9 & 206.6 & 523.5 & 231.1 & 238.1 \\
\hline$\geq 75$ & 2393.4 & 2383.7 & 1285.0 & 1370.7 & 1225.5 & 514.8 & 294.4 & 778 & 323.7 & 294.4 \\
\hline Total number & ○ 7145 & 5944 & 5644 & 4823 & 4214 & 2873 & 2390 & 2147 & 1981 & 1416 \\
\hline
\end{tabular}

*Including the brain; ${ }^{\circ}$ the number may be less than total due to missing data. 


\section{Discussion}

In this study, we reported the geo-epidemiological distribution of the TMPCs among Iranian men and women using populationbased cancer registries data. Iranian provinces were ranked based on the incidence of all types of cancers and the results were visually displayed on a map. Moreover, we showed geo-epidemiological distribution, spatial analysis of the incidence of TMPCs and clustered patterns on a provincial level using national data in Iran. In order to determine the tendency of neighbouring provinces to have similar incidence rates and formation clusters based on this correlation, we also investigated the spatial clustering and autocorrelation patterns of TMPCs (for both sexes) among the provinces of Iran.

In doing so, we found some controversial incidences for cancer of the ovary, breast and prostate in children less than 4 years old, which should be interpreted with caution. It was further shown that stomach cancer is the most prevalent cancer among Iranian men, with the highest incidences in Ardabil and Zanjan provinces. Previous studies have reported intake of high salt and opium

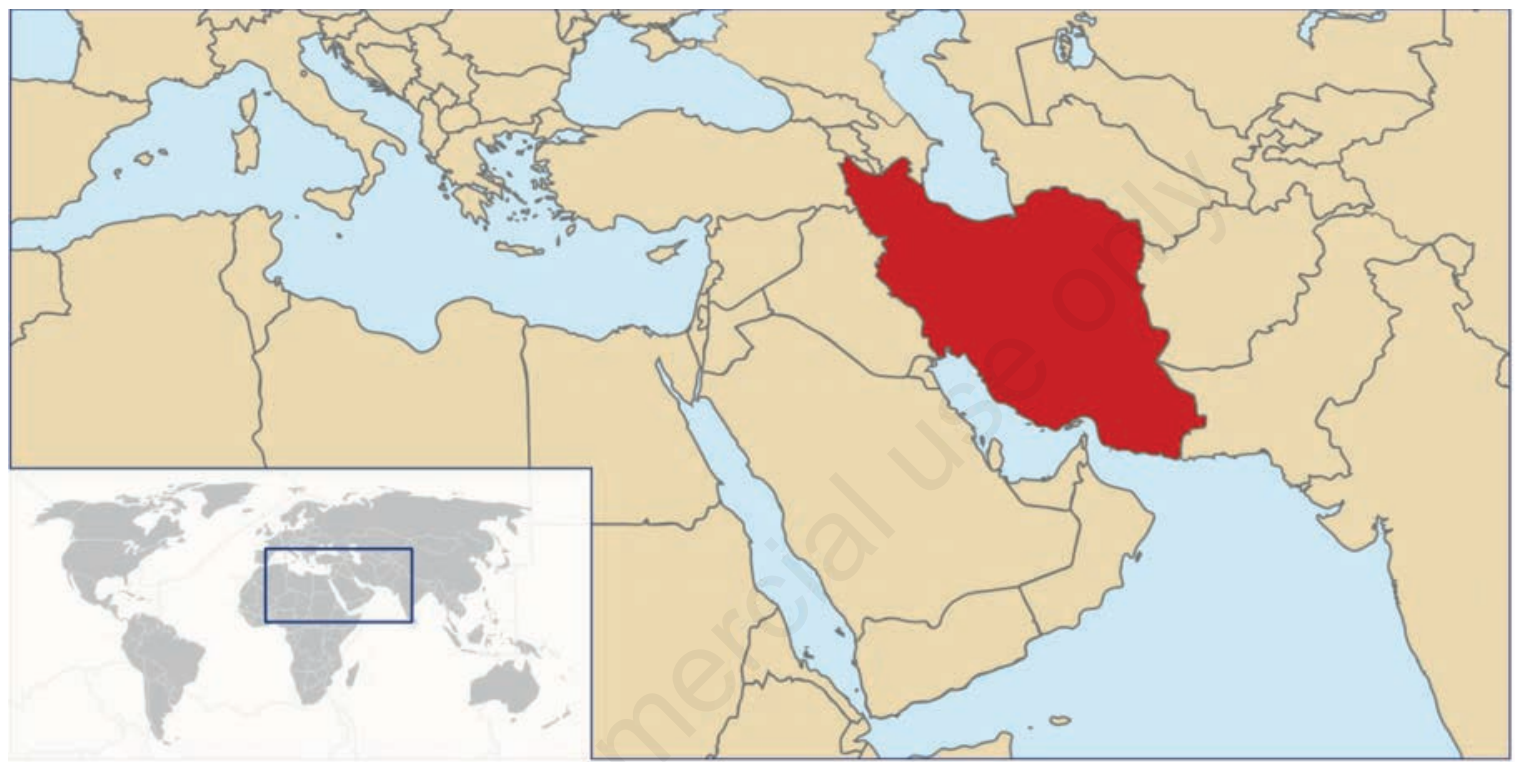

Figure 1. The geographical situation of Iran.

Table 3. Age-sex specific incidence rates (per 1,000,000) of the most common cancers in Iranian females 2014.

\begin{tabular}{lccccccccccc} 
Age group & Breast & Colorectal & Stomach & Thyroid & Leukaemia & Lung & Nervous system* & Oesophagus & Uterus & Ovary \\
$0-4$ & 0.2 & 0.2 & 0 & 0 & 43 & 0.9 & 16.3 & 0 & 0.3 \\
$5-9$ & 0 & 0.3 & 0.3 & 0.6 & 52.5 & 0.3 & 20.2 & 0 & 0 \\
\hline $10-14$ & 0.4 & 0.7 & 0 & 6.5 & 27.0 & 1.4 & 14.4 & 1.6 & 0 & 1.8 \\
$15-19$ & 2.2 & 3 & 3 & 18.3 & 33.7 & 2.6 & 12.7 & 2.2 & 0.4 & 8.2 \\
\hline $20-24$ & 18.1 & 8.2 & 5.7 & 63.1 & 26.9 & 4.1 & 19.3 & 0.6 & 2.2 & 11.4 \\
$25-29$ & 64.6 & 17.7 & 9.1 & 83.3 & 22.7 & 5.7 & 25.6 & 1 & 6.9 & 17.3 \\
\hline $30-34$ & 185.0 & 24.4 & 16.2 & 89.9 & 19.0 & 10.8 & 33.3 & 5.2 & 12.2 & 21.1 \\
$35-39$ & 370.5 & 47.0 & 30 & 101.9 & 23.1 & 8.4 & 29.4 & 11.5 & 26.5 & 28.6 \\
\hline $40-44$ & 658.2 & 88.0 & 48.8 & 116.5 & 36.6 & 26.6 & 49.2 & 17 & 51.4 & 48.1 \\
$45-49$ & 887.0 & 133.7 & 71.9 & 122.3 & 35.7 & 43.7 & 56.3 & 34.9 & 74.8 & 82.4 \\
\hline $50-54$ & 999.3 & 252.8 & 110.7 & 138.4 & 59.0 & 74.3 & 84.6 & 72.8 & 115.4 & 114.3 \\
$55-59$ & 935.3 & 324.3 & 186.8 & 110.1 & 85.1 & 111.9 & 85.1 & 110.1 & 177.3 & 113.1 \\
\hline $60-64$ & 951.2 & 437.2 & 317.1 & 122.5 & 119.4 & 173.7 & 108.5 & 179.1 & 210.1 & 144.2 \\
$65-69$ & 920.7 & 503.0 & 457.6 & 138.5 & 131.8 & 236.0 & 140.7 & 264.8 & 197.2 & 174.0 \\
\hline $70-74$ & 859.0 & 672.7 & 662.8 & 143.4 & 168.2 & 364.4 & 194.6 & 415.5 & 166.5 & 158.3 \\
$\geq 75$ & 758.9 & 854.0 & 1096.5 & 143.2 & 256.1 & 570.7 & 245.6 & 545.6 & 162 & 150.5 \\
\hline Total number & 13,116 & 4215 & 3343 & 2910 & 1879 & 1839 & 1779 & 1774 & 1725 & 1650 \\
\hline
\end{tabular}

*Including the brain; ${ }^{\circ}$ the number may be less than total due to missing data. 

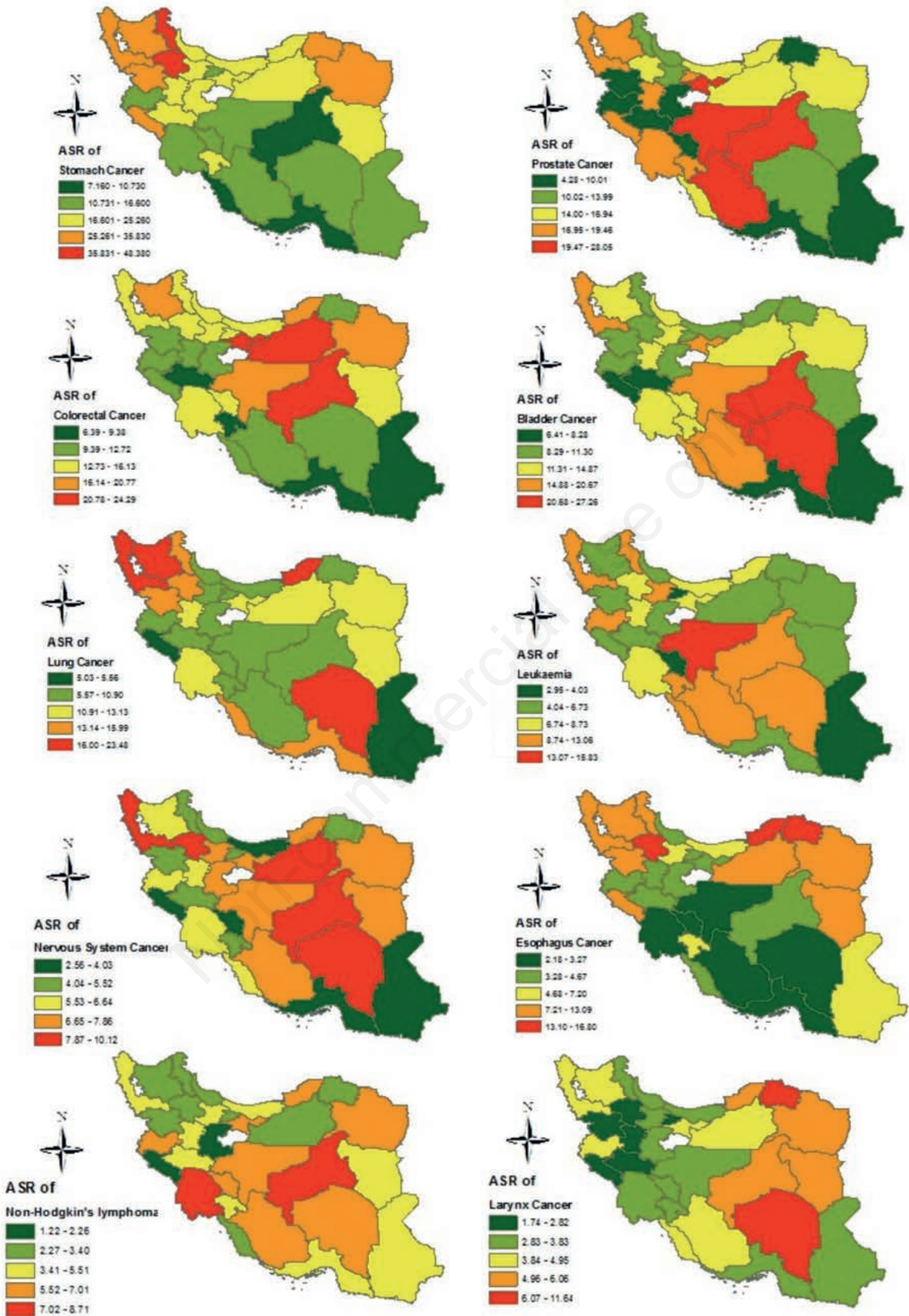

Figure 2. The geographic distribution in 2014 of the age-standardized incidence rates (ASR) per 100,000 population of the most common cancers in Iranian men. 

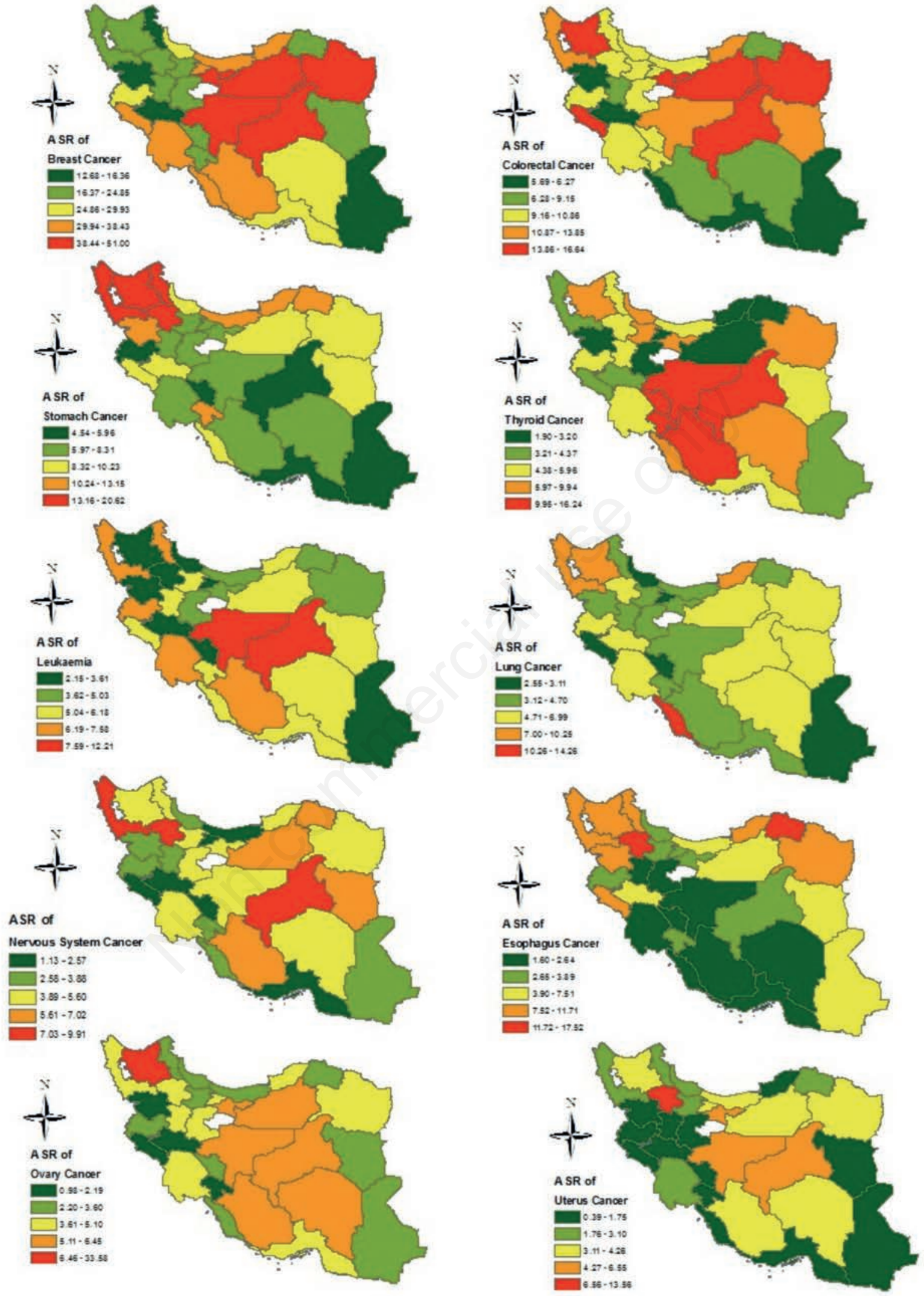

Figure 3. The geographic distribution in 2014 of the age-standardized incidence rates (ASR) per 100,000 population of the most common cancers in Iranian women. 

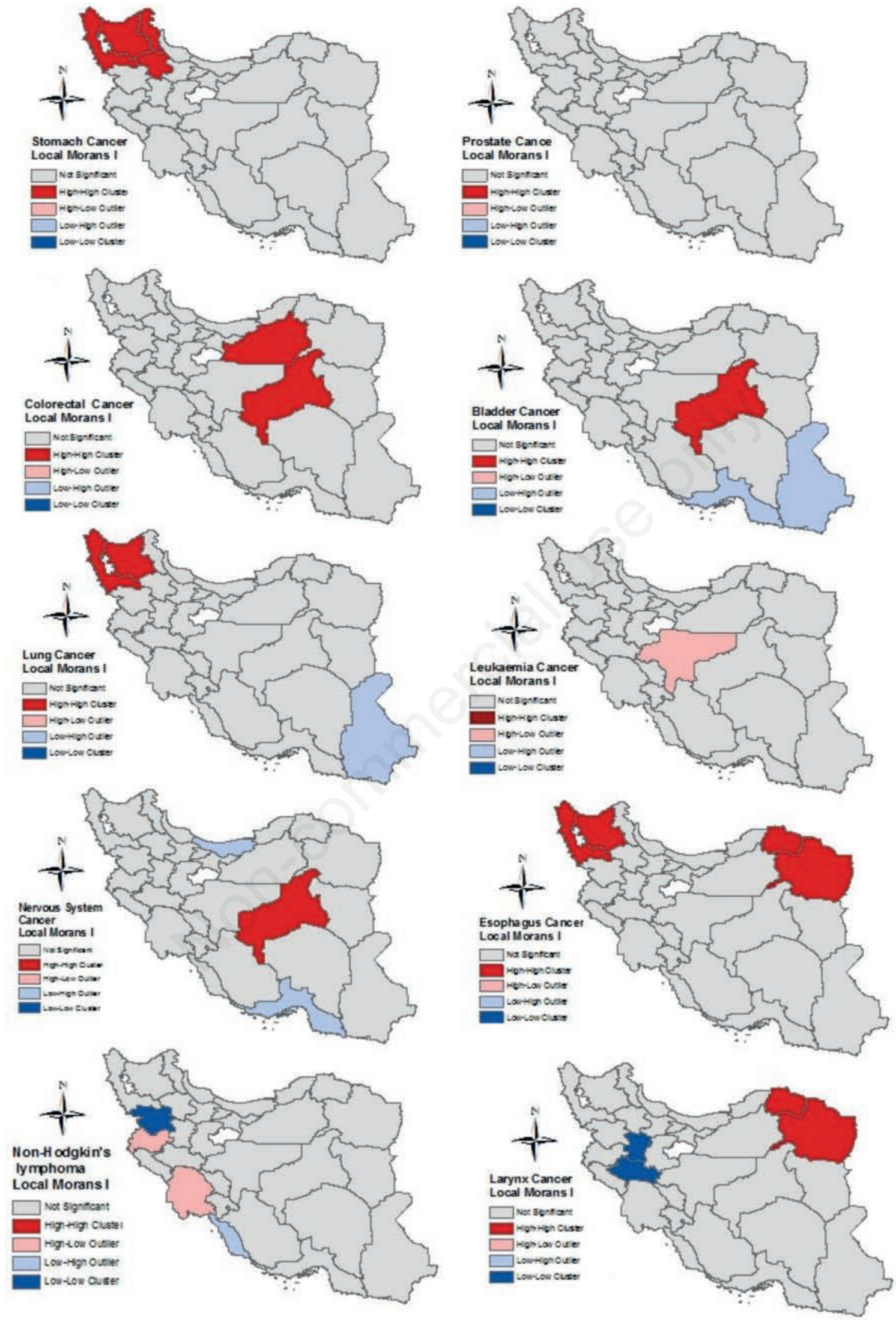

Figure 4. Spatial cluster patterns of the most common cancers in Iranian males in 2014 based on Moran's $I$. 

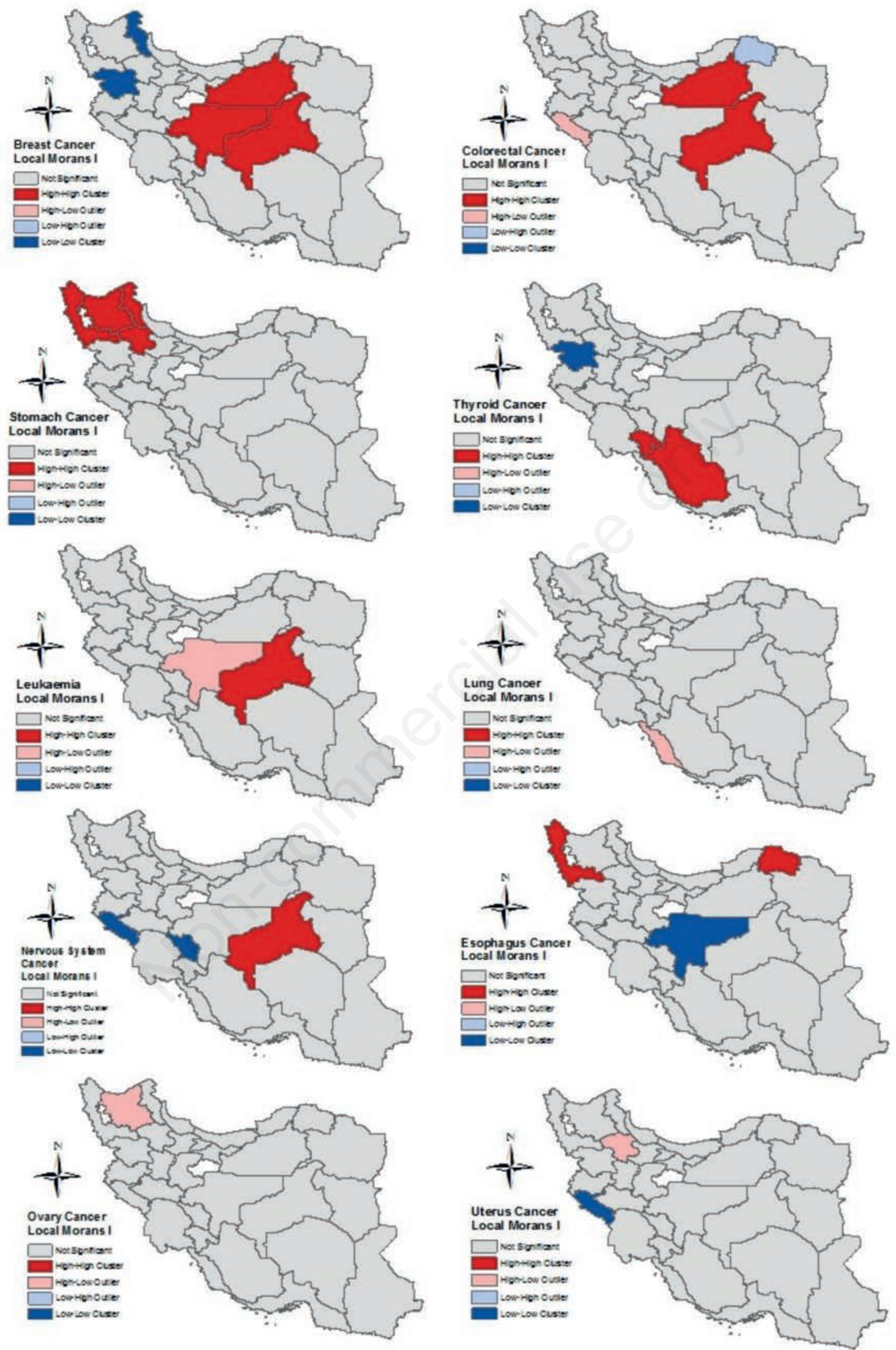

Figure 5. Spatial cluster patterns of the most common cancers in Iranian females in 2014 based on Moran's $I$. 
intake, as well as helicobacter infection, as risk factors of stomach cancer in these regions (Abdi et al., 2016; Yusefi et al., 2018). Several central provinces and most southern provinces had low incidence rates of this cancer. Notably, the highest incidence rates were in men and women aged 70 years and older.

Prostate and colorectal cancers were the second and third most prevalent cancers among Iranian men. It is noteworthy that the highest incidence rates of prostate cancer were in Yazd, Fars, and Tehran provinces. This cancer is particularly prevalent in developed countries (Baade et al., 2009), and a meta-analysis of 24 studies indicates that smoking is the most important risk factor (Huncharek et al., 2010). The incidence of prostate cancer has also been shown to be associated with the heavy metals such as copper, iron, and manganese (Karimi et al., 2012). With respect to colorectal cancer, we found its highest prevalence to be among Iranian men in Semnan, Tehran and Yazd provinces, respectively. A study was conducted in Khorasan-Razavi province in 2019 to identify the potential contributing factors of colorectal cancer. Accordingly, this study the incidence of colorectal cancer is significantly associated with a high obesity and low fibre intake level (Goshayeshi et al., 2019). Future studies along these lines will be very valuable to determine the environmental (Jacquez et al., 2003) and hereditary (Goshayeshi et al., 2017) contributing factors of colorectal cancer in the hotspot areas.

Larynx cancer is one of the least prevalent cancers among men, consequently ranked in the tenth place in our study. This cancer is mostly prevalent in North Khorasan Province. Based on the results of a study performed in 2016 in Iran, larynx cancer was considerably common in men, and the estimated age-standardized incidence rates were 21.6 and 3.2 per 100,000 in men and women, respectively (Mirzaei et al., 2016). In addition, a systematic review study in Iran confirms that larynx cancer is more common among men than women (Hassanipour et al., 2019).

In Iranian women, breast cancer is known as the most prevalent cancer (Roshandel et al., 2019), and women in Iran are affected at least a decade earlier compared to women in developed countries (Nematolahi \& Ayatollahi, 2017). Tehran, Yazd, Semnan, Khorasan-Razavi, and Isfahan provinces were found to have the highest incidence rates of breast cancer, in particular affecting women in the age group of 50-54 years and older. Other recent studies in Iran in 2020, though with a smaller sample size than our study, report the average age of women with breast cancer to be 54.4 years old (Dolatkhah et al., 2020).

Although Roshandel et al. (2019) report ovarian cancer as the 9th most prevalent cancer before uterus cancer among Iranian women, we found this cancer to be the 10th most prevalent cancer after uterus cancer in Iranian women. This can be due to subdivision of the codes used for the different components of the female genital organs, which might have caused a difference in the number of cancers of the internal organs. It seems therefore that the incidence rates of some cancers in some provinces should be interpreted with caution. Indeed, some southern and south-eastern provinces showed unusually low incidence rates of most cancers, which may be due to underreporting detected cancers by southeastern provinces or reporting of the cases of these provinces by other provinces. Because most patients are referred to other provinces due to the lack of diagnostic facilities in the south-eastern provinces.

In this study, we used exploratory spatial data analysis to determine spatial distribution patterns of cancers. The findings of this analysis can be helpful when investigating the potential effects of environmental factors as well as geographic and climatological agents on cancer incidence (Yomralioglu et al., 2009). As mentioned earlier, few studies have been conducted in this regard in Iran so far, and most of them were performed at the provincial level and on a small number of cancer types (Chamanparaa et al., 2015). After performing the analysis, spatial autocorrelation by Moran's $I$ showed significant clustering patterns in different regions for different cancers. In men, Moran's I showed stomach cancer as significantly clustered with high-high spatial patterns in West Azerbaijan, East Azerbaijan, Ardabil and Zanjan provinces. This indicates that the spatial distribution of this type of cancer tends to cluster across the north-western provinces of Iran. In other words, it seems that this clustering could be caused by common contributing risk factors across among these provinces.

For prostate cancer, there was no clustering pattern in any province. In 2006, a study performed in the UK showed a geographic variation in prostate cancer incidence across different counties, a variance possibly due to social, political, economic, health care and environmental differences (Gregorio et al., 2006). We also identified a high-high clustering pattern for colorectal cancer in men close to the centre of Iran in Semnan and Yazd provinces. Between 2007 and 2014, a study was conducted using Anselin Local Moran's I to identify clusters and outliers of colorectal cancer distribution in Hamadan province (Halimi et al., 2020). They demonstrated significant geographical disparities in colorectal cancer incidence with three significant clusters of both high and low incidence rates in Hamadan Province. Another study performed in Khorasan-Razavi Province identified three significantly high-low outlier areas as well as four significant low-high outliers (Goshayeshi et al., 2019).

In our study, lung cancer significantly clustered with a highhigh pattern in the Northwest of the country and a low-high outlier pattern for men in the Southeast. This pattern of outliers indicates that low-value areas are overlapped with high-value ones. Furthermore, we identified a high-low outlier region of leukaemia occurrence in Isfahan Province. A study performed in 2007 in Tehran showed some evidence of clustering of leukaemia in children in the central region of Tehran (Mosavi-Jarrahi et al., 2007). They showed that environmental factors may be strong risk factors for leukaemia.

In terms of breast cancer in women, we observed a low-low clustering pattern in Ardabil and Kurdistan provinces as well as a high-high one in the centre of Iran. Besides the known risk factors of breast cancer, previous studies have proven that cadmium, as a metalloestrogen, can be considered an environmental risk factor for breast cancer (Byrne et al., 2013). Similar to men, stomach cancer in women was significantly clustered with high-high patterns in north-western provinces. Moreover, female lung and ovary cancers only clustered as a high-low outlier in Bushehr and East Azerbaijan provinces, respectively.

We declare that several potential methodological limitations affected our study. This research was a registry-based study and this type of study have certain limitations in terms of the accuracy of data recording (Hailer, 2015). Another limitation was the use of the census population data (with a 5-year interval, instead of oneyear intervals) as dominator, to calculate the ASRs. The census is conducted in Iran every 5 years. If the census is conducted at oneyear intervals, more accurate demographic information may be available for health calculations. Incorrect recording of residence addresses of patients was another possible limitation of this study, in which the patient's place of diagnosis may be recorded as the 
patient's place of residence.

It is noteworthy that when recording patient information, many organizations and medical institutions do not record complete geographic data. Correspondingly, this can be considered as an important limitation when using GIS and spatial data for public health settings.

\section{Conclusions}

Complete and usable significant spatial and autocorrelation patterns of cancer incidence in Iran have been demonstrated. Analysis of the spatial distribution of cancers showed significant differences among different provinces. To investigate the environmental risk factors of cancers, researchers can consider the findings of this study in their future epidemiological studies. The findings of our study can also provide useful information for policymakers to plan appropriate screening programs and cancer surveillance.

\section{References}

Abdi E, Latifi-Navid S, Yazdanbod A, Zahri S, 2016. Helicobacter pylori babA2 positivity predicts risk of gastric cancer in Ardabil, a very high-risk area in Iran. Asian Pac J Cancer Prev 17:733-8.

Alireza S, Mehdi N, Ali M, Alireza M, Reza M, Parkin D, 2005. Cancer occurrence in Iran in 2002, an international perspective. Asian Pac J Cancer Prev 6:359.

Baade PD, Youlden DR, Krnjacki LJ, 2009. International epidemiology of prostate cancer: Geographical distribution and secular trends. Mol Nutr Food Res 53:171-84.

Bafandeh Y, Farhang S, 2009. Subsite distribution of gastric cancer in an area of high prevalence-northwest Iran. J Epidemiol 19:202-5.

Bergquist R, Manada S, 2019. The world in your hands: GeoHealth then and now. Geospat Health 14:779.

Bray F, Ferlay J, Soerjomataram I, Rebecca L, Siegel RL, Torre LA, Jemal A, 2018. Global cancer statistics 2018: GLOBOCAN estimates of incidence and mortality worldwide for 36 cancers in 185 countries. Ca Cancer J Clin 68:394-424.

Byrne C, Divekar SD, Storchan GB, Parodi DA, Martin MB, 2013. Metals and breast cancer. J Mammary Gland Biol Neoplasia 18:63-73.

Chamanparaa P, Moghimbeigi A, Faradmal J, Poorolajal J, 2015. Exploring the spatial patterns of three prevalent cancer latent risk factors in Iran; using a shared component model. IJER 2:68-77.

Global Burden of Disease Cancer Collaboration, 2019. Global, regional, and national cancer incidence, mortality, years of life lost, years lived with disability, and disability-adjusted life-years for 29 cancer groups, 1990 to 2017: a systematic analysis for the Global Burden of Disease Study. JAMA Oncol 5:1749-68.

Dolatkhah R, Somi MH, Jafarabadi MA, Hosseinalifam M, Sepahi S, Belalzadeh M, Nezamdoust M, Dastgiri S, 2020. Breast cancer survival and incidence: 10 years cancer registry data in the northwest. Int J Breast Cancer 2020:1963814.

Fritz AG, 2013. International classification of diseases for oncology: ICD-O. World Health Organization, Geneva, Switzerland.
Goshayeshi L, Khooiee A, Ghaffarzadegan K, Khorram MR, Bishehsari F, Hoseini B, Rezayat KA, Esmaeilzadeh A, Mozaffari HM, Ghanayee O, 2017. Screening for Lynch syndrome in cases with colorectal carcinoma from Mashhad. AIM 20:332-7.

Goshayeshi L, Pourahmadi A, Ghayour-Mobarhan M, Hashtarkhani S, Karimian S, Dastjerdi R, Eghbali B, Seyfi E, Kiani B, 2019. Colorectal cancer risk factors in north-eastern Iran: A retrospective cross-sectional study based on geographical information systems, spatial autocorrelation and regression analysis. Geospat Health 14:793.

Gregorio DI, Samociuk H, DeChello L, Swede H, 2006. Effects of study area size on geographic characterizations of health events: prostate cancer incidence in Southern New England, USA, 1994-1998. Int J Health Geograph 5:8.

Hailer NP, 2015. Orthopedic registry research-limitations and future perspectives. Acta Orthop 86:1.

Halimi L, Bagheri N, Hoseini B, Hashtarkhani S, Goshayeshi L, Kiani B, 2020. Spatial analysis of colorectal cancer incidence in Hamadan Province, Iran: a retrospective cross-sectional study. Appl Spat Analy Policy 13:293-303.

Hao Y, Landrine H, Jemal A, Ward KC, Bayakly AR, Young JL, Flanders WD, Ward EM, 2011. Race, neighbourhood characteristics and disparities in chemotherapy for colorectal cancer. JECH 65:211-17.

Hassanipour S, Delam H, Nikbakht HA, Abdzadeh E, Salehiniya H, Arab-Zozani M, Ghaem H, 2019. The incidence of laryngeal cancer in Iran: a systematic review and meta-analysis. CEGH 7:457-63.

Huncharek M, Haddock KS, Reid R, Kupelnick B, 2010. Smoking as a risk factor for prostate cancer: a meta-analysis of 24 prospective cohort studies. AJPH 100:693-701.

Jacquez GM, Greiling DA, 2003. Geographic boundaries in breast, lung and colorectal cancers in relation to exposure to air toxics in Long Island, New York. Int J Health Geograph 2:4.

Kamangar F, Dores GM, Anderson WF, 2006. Patterns of cancer incidence, mortality, and prevalence across five continents: defining priorities to reduce cancer disparities in different geographic regions of the world. J Clin Oncol 24:2137-50.

Karimi G, Shahar S, Homayouni N, Rajikan R, Bakar NF, Othman MS, 2012. Association between trace element and heavy metal levels in hair and nail with prostate cancer. APJCP 13:4249-53.

Mirzaei M, Hosseini SA, Ghoncheh M, Soheilipour M, Soltani S, Soheilipour F, Salehiniya H, 2016. Epidemiology and trend of head and neck cancers in Iran. Global J Health Sci 8:189.

Mosavi-Jarrahi A, Moini M, Mohagheghi MA, Alebouyeh M, Yazdizadeh B, Shahabian A, Nahvijo A, Alizadeh R, 2007. Clustering of childhood cancer in the inner city of Tehran metropolitan area: a GIS-based analysis. Int J Hyg Environ Health 210:113-9.

Mousavi SM, Davanlou M, Gouya MM, Hajsadeghi N, Ramazani R, Seddighi Z, 2008. Cancer incidence and mortality in Iran. Ann Oncol 20:556-63.

Mousavi SM, Gouya MM, Ramazani R, Davanlou M, Hajsadeghi N, Seddighi Z, 2009. Cancer incidence and mortality in Iran. Ann Oncol 20:556-63.

Nematolahi S, Ayatollahi MT, 2017. A comparison of breast cancer survival among young, middle-aged, and elderly patients in southern Iran using Cox and empirical Bayesian additive hazard models. Epidemiol Health 39.

Roshandel G, Ghanbari-Motlagh A, Partovipour E, Salavati F, 
Hasanpour-Heidari S, Mohammadi G, Khoshaabi M, Sadjadi A, Davanlou M, Tavangar SM, 2019. Cancer incidence in Iran in 2014: results of the Iranian National Population-based Cancer Registry. Cancer Epidemiol 61:50-8.

Scholten H, de Lepper MJ, 1991. The benefits of the application of geographical information systems in public and environmental health. World Health Stat Q 44:160-70.

Segi M, 1960. Cancer mortality for selected sites in 24 countries (1950-57). Department of Public Health, Tohoku University of Medicine, Sendai, Japan.
The Lancet, 2018. GLOBOCAN 2018: counting the toll of cancer. Lancet 392:985.

Yomralioglu T, Colak EH, Aydinoglu AC, 2009. Geo-relationship between cancer cases and the environment by GIS: A case study of Trabzon in Turkey. Int J Environ Res Public Health 6:3190-204.

Yusefi A, Lankarani KB, Bastani P, Radinmanesh M, Kavosi Z, 2018. Risk factors for gastric cancer: a systematic review. APJCP 19:591. 